\title{
MEASURING THE IONIZATION OF O STAR WINDS
}

\author{
Luciana Bianchi, ${ }^{1,2,3}$ J. B. Hutchings, ${ }^{1,4}$ Alex W. Fullerton, ${ }^{2,5}$ Donald C. Morton, ${ }^{1,4}$ Raymond G. Ohl, ${ }^{2}$ \\ George Sonneborn, ${ }^{6}$ Derck Massa, ${ }^{7}$ K. R. Brownsberger, ${ }^{8}$ and Allan J. Willis ${ }^{9}$ \\ Received 2000 March 21; accepted 2000 June 7; published 2000 July 14
}

\begin{abstract}
We present an analysis of wind line profiles from Far Ultraviolet Spectroscopic Explorer (FUSE) spectra of two 07 supergiants in the Large and Small Magellanic Clouds (Sk $-67^{\circ} 111$ and AV 232, respectively). Model fits yield the column densities of S IV, S VI, P IV, P V, N III, and N IV, providing the first direct measurement of the ionization balance in stellar winds. The ratios of $\mathrm{S}$ IV/S VI and P IV/P V are consistently lower in the LMC star. IUE and Hubble Space Telescope archival spectra are also used to measure $\mathrm{N}$ IV and $\mathrm{N} v$, but the much higher optical depth makes the $\mathrm{N} v$ measurements inconclusive. The velocity and optical depth distributions in the wind are qualitatively similar between the two stars, when scaled to their terminal velocities. The terminal velocities are different, with AV 232 being lower (as found previously in SMC stars and linked to lower metallicity). These are the first results from a program to investigate wind ionization and velocity structure among hot stars in local galaxies, and they demonstrate the higher accuracy in measuring column densities of less abundant ions, such as phosphorus and sulfur, observable in the FUSE range.
\end{abstract}

Subject headings: Magellanic Clouds - stars: early-type — stars: winds, outflows - ultraviolet: stars

\section{INTRODUCTION}

Supersonic winds of hot stars are revealed by conspicuous $\mathrm{P}$ Cygni profiles in the strongest transitions of the most abundant ions/species, which are found below $2000 \AA$. The wavelength region accessible to IUE and Hubble Space Telescope (HST; longward of $1200 \AA$ ) contains strong resonance lines of $\mathrm{C}$ IV, $\mathrm{N}$ v, and Si IV. Observations of these lines in stars in a variety of environments established a strong influence of the metal abundance on the wind terminal velocity (e.g., Bianchi \& Scuderi 1999 and references therein; Bianchi, Hutchings, \& Massey 1996; Prinja \& Crowther 1998). However, these lines are often saturated and alone are not sufficient to determine the mass-loss rate and the ionization structure of the wind. The Far Ultraviolet Spectroscopic Explorer (FUSE; Moos et al. 2000) range (905-1187 $\AA$ ) includes a greater spread of species and ionization states, allowing us to disentangle abundances and wind ionization structure for hot stars.

We present early FUSE observations of two O7 stars, Sk $-67^{\circ} 111$ in the Large Magellanic Cloud (LMC) and AV 232 (= Sk 80) in the Small Magellanic Cloud (SMC), and compare the ionization of their winds. These are part of a FUSE team program to investigate hot star winds in different local environments. FUSE observations of similar Galactic stars have not been obtained yet, so our comparison is limited to the LMC

\footnotetext{
${ }^{1}$ Observer with the NASA-CNES-CSA Far Ultraviolet Spectroscopic Explorer (FUSE), which is operated by Johns Hopkins University under NASA contract NAS5-32985.

${ }^{2}$ Center for Astrophysical Sciences, Johns Hopkins University, Department of Physics and Astronomy, 3400 North Charles Street, Baltimore, MD 21218.

${ }^{3}$ Osservatorio Astronomico di Torino, Pino Torinese (TO), Italy.

${ }^{4}$ Herzberg Institute of Astrophysics, National Research Council of Canada, 5071 West Saanich Road, Victoria, BC V8X 4M6, Canada.

${ }^{5}$ Department of Physics and Astronomy, University of Victoria, P.O. Box 3055, Victoria, BC V8W 3P6, Canada.

${ }^{6}$ Laboratory for Astronomy and Solar Physics, NASA Goddard Space Flight Center, Code 681.0, Greenbelt, MD 20771.

${ }^{7}$ Raytheon Information Technology and Scientific Services, Code 681.0, NASA Goddard Space Flight Center, Greenbelt, MD 20771.

${ }^{8}$ Center for Astrophysics and Space Astronomy, University of Colorado, Campus Box 389, Boulder, CO 80309.

${ }^{9}$ Department of Physics and Astronomy, University College London, Gower Street, London WC1E 6BT, England, UK.
}

and SMC. Copernicus observations reported by McCluskey, Kondo, \& Morton (1975) and Snow \& Morton (1976) of the O7 Ia:fp eclipsing binary star $29 \mathrm{C}$ Ma (= UW C Ma = HD 57060) show very strong $S$ IV and $P$ v P Cygni profiles. We reexamined the archival Copernicus data, but found there is no significant signal at $\mathrm{S}$ VI and P IV. IUE spectra with better signal than the Copernicus data for $\mathrm{C}$ IV and $\mathrm{N} v$ were analyzed by Chlebowski \& Garmany (1991) and by Bianchi (1982), the latter finding values of the terminal velocity from $V_{\infty}=1640$ to $1800 \mathrm{~km} \mathrm{~s}^{-1}$. However, we note that $29 \mathrm{C}$ Ma is not a good Galactic comparison because it is variable and peculiar (O7 Ia:fpvar to O9 I) and has a hot companion that causes complex effects of colliding winds (e.g., Chlebowski, Harnden, \& Sciortino 1989; Bianchi 1982).

The early FUSE spectra used in this Letter do not have the final accuracy that we expect to reach in future observations, but they are quite adequate to demonstrate the potential of FUSE observations for the analysis of broad wind lines. The line profiles are modeled with the Sobolev plus exact integration (SEI) code $(\S 3)$ to derive the optical depth of the S IV, $\mathrm{S}$ VI, $\mathrm{P}$ IV, $\mathrm{P}$ V, $\mathrm{N}$ III, and $\mathrm{N}$ IV ions.

AV 232 was observed by the Hopkins Ultraviolet Telescope (HUT; Davidsen et al. 1992) during the Astro-2 mission. The HUT spectrum, shown by Walborn et al. (1995b), has a resolution of $3 \AA$, which is insufficient to detect and remove the effects of interstellar absorption components. The higher resolution of FUSE shows that this contamination is very severe.

\section{THE PROGRAM STARS AND THE OBSERVATIONS}

Table 1 lists relevant information on the stars and our data. All data were taken in the $30^{\prime \prime} \times 30^{\prime \prime}$ (LWRS) aperture. The "X" data sets were obtained in 1999 September as part of the Early Release Observation program and include only the LiF1 channel. The "I" data sets were obtained in 1999 October during the in-orbit checkout phase as mirror alignment tests. The stars were swept through the apertures during the exposures, resulting in total exposure times in different channels ranging from 2.7 to $4.9 \mathrm{ks}$ for $\mathrm{Sk}-67^{\circ} 111$ and from 1.2 to $4.9 \mathrm{ks}$ for AV 232. The standard data pipeline cannot be used to extract these spectra, and special processing was required to extract 
TABLE 1

Program Stars and FUSE Data

\begin{tabular}{|c|c|c|c|c|c|c|c|}
\hline \multirow[b]{2}{*}{ STAR } & \multicolumn{2}{|c|}{ Spectral Type } & \multirow[b]{2}{*}{$V$} & \multicolumn{2}{|c|}{ LSR VELOCITY } & \multirow[b]{2}{*}{ FUSE Data Sets } & \multirow[b]{2}{*}{$\begin{array}{c}V_{\infty} \\
\left(\mathrm{km} \mathrm{s}^{-1}\right)^{\mathrm{a}}\end{array}$} \\
\hline & Value & Reference & & $\begin{array}{c}\text { Value } \\
\left(\mathrm{km} \mathrm{s}^{-1}\right)\end{array}$ & Reference & & \\
\hline LMC Sk $-67^{\circ} 111 \ldots \ldots$ & O7 Ib(f) & 1 & 12.57 & 250 & 2 & X0200101, I8010901 & $1800 \pm 100$ \\
\hline SMC AV $232 \ldots \ldots \ldots \ldots$ & $\mathrm{O} 7 \mathrm{Iaf}^{+}$ & 3 & 12.36 & 164 & 3 & X0200201, I8010801 & $1400 \pm 100$ \\
\hline
\end{tabular}

${ }^{\text {a }}$ Values taken from this Letter.

REFERENCES. - (1) Fitzpatrick 1988; (2) Hutchings 1982; (3) Walborn et al. 1995a.

the useful data. Details of spectral extractions and final quality assessment are given in a companion Letter (Massa et al. 2000). For the I data, independent extractions by different authors resulted in data with negligible differences. All data were taken prior to telescope focusing, and the spectral resolution ranges over 12,000-15,000. This is quite sufficient for our purpose of analyzing very broad stellar lines. In this Letter, we model only line profiles (flux relative to the local continuum), so uncertainties in the absolute flux calibration are not a concern. The uncertainty in the wavelength scale is not well established in these early data. Small-scale nonlinearities might be present which would affect measurements of very narrow lines but not the fits of broad wind profiles. To obtain a conservative (when applied to wind velocities) estimate of the uncertainty, we measured identified interstellar lines near the stellar lines of interest. We found the accuracy to be comparable to the resolution for most lines and worse by a factor of 2 around the S IV and $\mathrm{S}$ VI lines.

We also analyzed $\mathrm{N}$ IV and $\mathrm{N} \mathrm{v}$ lines from IUE archive spectra of Sk $-67^{\circ} 111$ (SWP 10991 and SWP 52745 combined) and from HST-Faint Object Spectrograph (FOS) archive spectra (Y14M05) of AV 232.

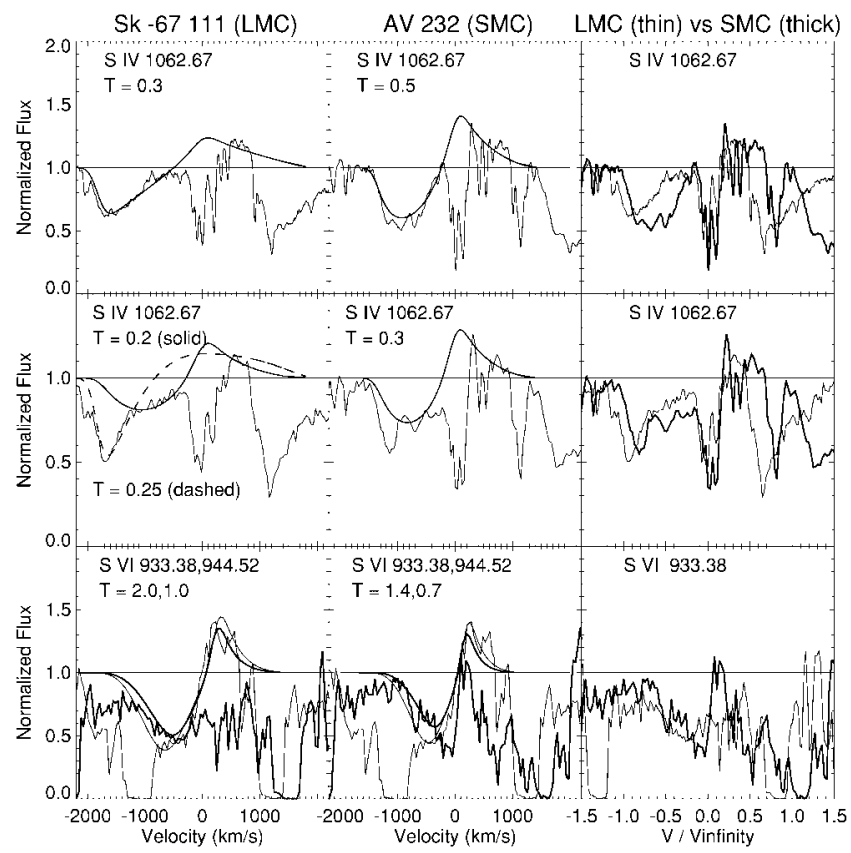

FIG. 1. - Left and middle columns: Model fits to the line profiles of S IV and S VI. In the S IV doublet the $\lambda 1062.66$ transition is chosen because it is the less blended (top: $\mathrm{X}$ data; middle: I data). For the $\mathrm{S}$ vI doublet, both lines are plotted: $\lambda 944.52$ (thick line) and $\lambda 933.38$ (thin line). Note the severe interstellar line absorptions. In the right column, the observed profiles of the two stars are compared, each normalized in width to its wind terminal velocity derived by the model. In this display, interstellar components are displaced by different amounts scaled by the terminal velocity ratio, but the similarity of the stellar features can be appreciated.

\section{LINE MODELING}

The FUSE range contains resonance lines of both S VI and $\mathrm{S}$ IV ions and both $\mathrm{P}$ IV and $\mathrm{P} v$. These lines allow the first direct, empirical comparison of the wind ionization among two stars in different galaxies, without assumption or model derivation of the element abundances.

To include an abundant element from the CNO cycle, we also analyzed nitrogen lines. The $\mathrm{N}$ III resonance line $\lambda 989.799$ is blended with two lines from a slightly excited level: $\lambda 991.577$ of almost equal strength and $\lambda 991.511$ of negligible intensity. For N IV, only excited transitions are available $-\lambda 955.334$ in the FUSE range and $\lambda 1718.550$ in the IUE/FOS range. Finally, the resonance doublet of $\mathrm{N} v \lambda \lambda 1238.821,1242.804$ from the IUE/FOS data was also modeled, although the uncertainties increase rapidly with high optical depths.

The observed P Cygni profiles are modeled with the SEI method (Lamers, Cerruti-Sola, \& Perinotto 1987) using the parameterization adopted by Bianchi, Vassiliadis, \& Dopita (1997). The velocity law in the wind is expressed as

$$
w(x)=\frac{v(x)}{V_{\infty}}=w_{0}+\left(1-w_{0}\right)\left(1-\frac{1}{x}\right)^{\gamma},
$$

where $x=r / R_{*}, R_{*}$ is the photospheric radius, and $w_{0}$ is the initial velocity at the base of the wind. The optical depth of the transition is expressed as

$$
\tau(w)=\left(\frac{T}{I}\right)\left(\frac{w}{w_{1}}\right)^{\alpha_{1}}\left[1-\left(\frac{w}{w_{1}}\right)^{1 / \gamma}\right]^{\alpha_{2}},
$$

where $w_{1}$ is the maximum velocity shift in the line profile as a fraction of the full terminal velocity, $T$ is the total optical depth, and

$$
I=w_{1} \int_{w_{0} / w_{1}}^{1} y^{\alpha_{1}}\left(1-y^{1 / \gamma}\right)^{\alpha_{2}} d y .
$$

The normalization factor $I$ ensures that $T_{i}$ (optical depth of a given line of the ion $i$ ) is proportional to the column density $N_{i}$ of the given ion, $T_{i} \propto f \lambda N_{i} V_{\infty}^{-1}$ (see, e.g., Bianchi et al. 1994). Photospheric absorption has not been included in the fits shown. Inclusion of a photospheric profile will visually improve the match to the observed profile around zero velocities, but would not affect the resulting wind parameters.

The results are shown in Figures 1-3. The I data contain all four channels and thus include both S VI and S IV lines, while only S IV is included in the X data. Because the stellar line profiles show variability in time, as discussed by Massa et al. (2000), we use only results from the I data for comparison of the two ions. However, we also fit the $\mathrm{S}$ Iv lines from the $\mathrm{X}$ data to quantify the variability of the optical depth (Fig. 1). These profiles appear to have a stronger absorption, and 




FIG. 2.-SEI fits of $P \vee \lambda 1117.98$ and $P$ IV $\lambda 950.66$. The second is severely contaminated by interstellar absorptions. The solid and dotted lines show the range of allowable models given the interstellar contamination. Columns are as in Fig. 1.

smoother profile, than the I data. The S IV profiles in the I data show several narrow dips, possibly due to wind clumps. If the observed narrow dips are produced by strong inhomogeneities in the stellar wind, the column density would be higher than in the smooth fit (see Fig. 1 [dotted model] and Table 2).

The profiles of S vi $\lambda \lambda 933.38,944.52$ are heavily absorbed by interstellar lines (molecular hydrogen in particular), which makes it difficult to identify good portions of the profile to model. Because the lines arise from the same multiplet, their optical depths should be in the ratio of their oscillator strengths, with all other model parameters (optical depth law, turbulence, and velocity law) being the same. By forcing the total optical depth to be in the ratio $2: 1$, we derived the best fits shown in Figure 1, where both $\mathrm{S}$ vi blue and red components are plotted, each in velocity scale relative to its appropriate rest wavelength. In this way it is easier to identify the bona fide (stellar) parts of each profile.

\section{CONCLUSIONS}

SEI profile fitting provides a direct measure of the total optical depth of each transition, which is proportional to the total column density of the ion in the wind (e.g., Bianchi et al. 1994). Our results thus yield the column density ratio of

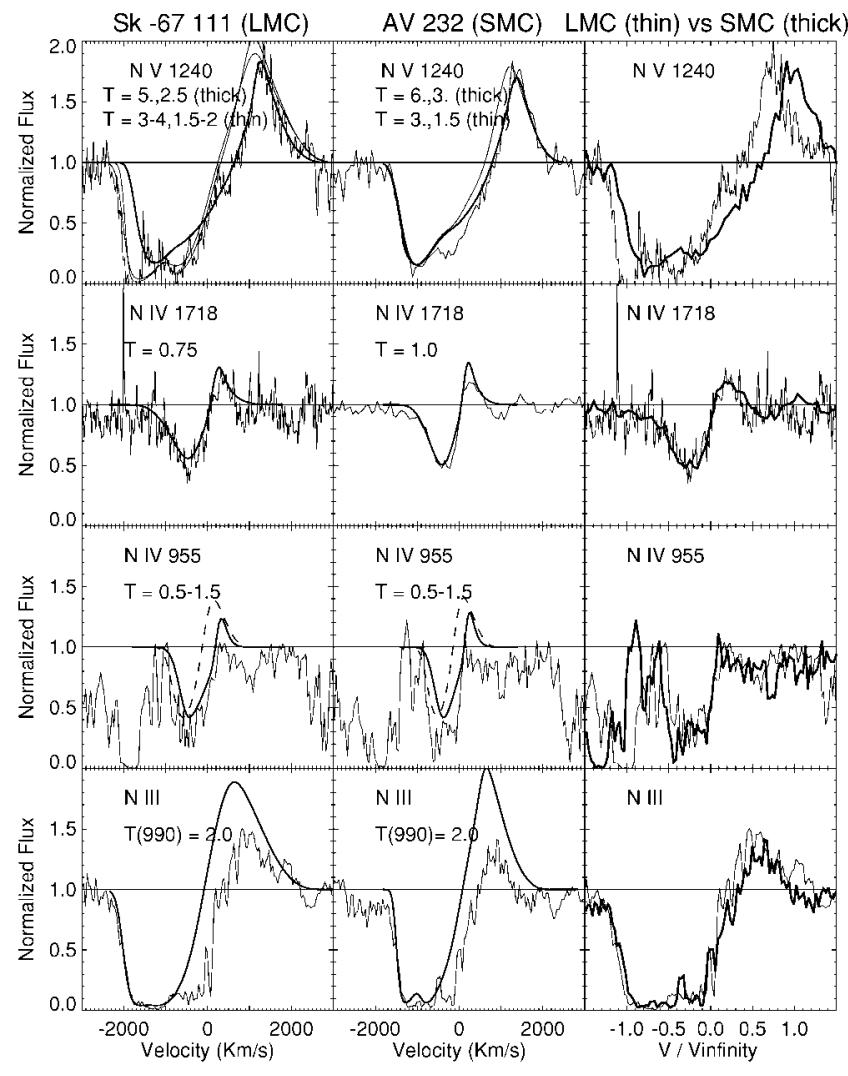

FIG. 3.-SEI fits of the nitrogen lines; columns as in Fig. 1. The top two lines are from IUE and FOS data (Sk $-67^{\circ} 111$ and AV 232, respectively); the FOS data have lower resolution and higher signal-to-noise ratio. The two bottom lines (N IV and $\mathrm{N}$ III) are from the FUSE data. Both N IV transitions are from an excited level. The $\mathrm{N}$ III feature contains two equally strong lines: $\lambda 989.799$ from the ground state and $\lambda 991.577$ from an excited level.

different ions of the same element, which can be used to constrain atmosphere and wind models. The results are given in Table 2. The ratios of $N_{i}(\mathrm{~S} \mathrm{IV}) / N_{i}(\mathrm{~S}$ vI $)$ and $N_{i}(\mathrm{P}$ IV $) / N_{i}(\mathrm{P} \mathrm{v})$ are smaller in Sk $-67^{\circ} 111$ than in AV 232.

For small optical depths $(\leq 2)$, the uncertainty from the model fit is small: an optical depth value different by \pm 0.05 would create a very obvious mismatch to the data. For some lines, a source of uncertainty is the location of the true stellar profile and local continuum, due to the strong interstellar absorptions in the FUSE range. To derive the uncertainties of the values quoted in Table 2, we varied all fit parameters within acceptable ranges, as well as the placement of the local continuum. Re-

TABLE 2

Column Densities from SEI Line Modeling

\begin{tabular}{|c|c|c|c|c|c|c|c|c|}
\hline \multirow[b]{2}{*}{ Transition } & \multirow[b]{2}{*}{ DATA } & \multicolumn{2}{|r|}{$f$} & \multicolumn{2}{|c|}{ Sk $-67^{\circ} 111$} & \multicolumn{3}{|c|}{ AV 232} \\
\hline & & Value & Reference & $T$ & $\begin{array}{c}N_{i} \\
\left(\times 10^{15} \mathrm{~cm}^{-2}\right)\end{array}$ & & $T$ & $\begin{array}{c}N_{i} \\
\left(\times 10^{15} \mathrm{~cm}^{-2}\right)\end{array}$ \\
\hline \multirow[t]{2}{*}{ S IV $\lambda 1062.664 \ldots \ldots}$. & FUSE X & 0.0593 & 1 & $0.30 \pm 0.05$ & $3.2 \pm 0.5$ & 0.5 & \pm 0.05 & $4.2 \pm 0.4$ \\
\hline & FUSE I & 0.0593 & 1 & $0.20 \pm 0.05$ & $2.2 \pm 0.5^{\mathrm{a}}$ & 0.3 & \pm 0.05 & $2.5 \pm 0.4$ \\
\hline$S$ VI $\lambda 933.378$ & FUSE I & 0.439 & 2 & $2.00 \pm 0.10$ & $3.3 \pm 0.2$ & 1.4 & \pm 0.1 & $1.8 \pm 0.1$ \\
\hline$P$ IV $\lambda 950.657$ & FUSE I & 1.56 & 3 & $0.75 \pm 0.25$ & $0.3 \pm 0.1$ & 3.0 & \pm 1.0 & $1.1 \pm 0.4$ \\
\hline $\mathrm{P} \vee \lambda 1117.977$. & FUSE I & 0.473 & 2 & $0.45 \pm 0.05$ & $0.6 \pm 0.1$ & 0.5 & \pm 0.05 & $0.5 \pm 0.05$ \\
\hline N III $\lambda 989.799$. & FUSE I & 0.123 & 4 & $2.00 \pm 0.50$ & $11.1 \pm 2.8$ & 2.00 & \pm 0.50 & $8.7 \pm 2.2$ \\
\hline $\mathrm{N} \mathrm{IV}^{\star} \lambda 955.335$ & FUSE I & 0.133 & 4 & $1.00 \pm 0.50$ & $5.3 \pm 2.7$ & 1.00 & \pm 0.50 & $4.2 \pm 2.1$ \\
\hline $\mathrm{N} \mathrm{IV}^{\star} \lambda 1718.551 \ldots \ldots$ & $I U E / \mathrm{FOS}$ & 0.171 & 4 & $0.75 \pm 0.10$ & $1.7 \pm 0.2$ & 1.00 & \pm 0.10 & $1.8 \pm 0.2$ \\
\hline $\mathrm{N} v \lambda 1238.821 \ldots \ldots$ & IUE/FOS & 0.156 & 4 & $4.00 \pm 1.00$ & $14.0 \pm 3.5$ & 4.50 & \pm 1.50 & $12.3 \pm 4.1$ \\
\hline
\end{tabular}

${ }^{a}$ Using $T=0.25$, which includes the narrow absorption component, $N_{i}(\mathrm{~S}$ IV) becomes $2.69 \pm 0.54$.

References. - (1) Reistad \& Engstrom 1989; (2) Morton 1991; (3) Froese-Fischer \& Godefroid 1982; (4) Wiese, Fuhr, \& Deters 1996. 
gardless of the model results and their accuracy, a visual inspection of the line profiles normalized to the wind terminal velocities (see right column, Figs. 1-3) reveals a remarkable similarity between the two stars. It also reveals that in the LMC star S IV and P IV are weaker, S VI is stronger, and P V is in an intermediate situation, which suggests a correlation of the differences with the ionization potentials. These qualitative differences are quantified by the measurements of the column densities in Tables 2 and 3.

The SEI fits also yield a measurement of the wind terminal velocity. The best values from all the lines analyzed in this work are given in Table 1: $V_{\infty}=1400 \mathrm{~km} \mathrm{~s}^{-1}$ for AV 232 and $V_{\infty}=1800 \mathrm{~km} \mathrm{~s}^{-1}$ for $\mathrm{Sk}-67^{\circ} 111$. The wind velocity derived by line fitting is more accurate than values estimated simply from the edge of the absorption, since microturbulence in the outflow is taken into account (a turbulence of $0.1 V_{\infty}$ was found from our fits, which is typical for this type of star). Previous determinations of wind velocities from IUE spectra (e.g., Patriarchi \& Perinotto 1992; $V_{\infty}=2090 \pm 220 \mathrm{~km} \mathrm{~s}^{-1}$ for Sk $-67^{\circ} 111$ and $V_{\infty}=1410 \pm 200 \mathrm{~km} \mathrm{~s}^{-1}$ for AV 232) are consistent with our results (noting that we have removed the intrinsic velocity of each star) and the respective uncertainties. Haser et al. (1994) found $V_{\infty}=1400 \mathrm{~km} \mathrm{~s}^{-1}$ for AV 232 from a similar SEI modeling of different lines.

In summary, we have measured column densities of $S$ IV, $\mathrm{S}$ VI, $\mathrm{P}$ IV, $\mathrm{P}$ V, $\mathrm{N}$ III, $\mathrm{N}$ IV, and $\mathrm{N} v$ in the winds of two $\mathrm{O} 7$ supergiants in the LMC and SMC, respectively. The ratio between ions of sulfur and phosphorus is different in the two stars. This might be due to the different luminosity and/or different density in the wind, which can be expected if the mass-loss rates are different (because the metallicities are different). The wind reaches a significantly higher velocity in the LMC star, consistent with the globally different metallicity and with previous findings. However, the optical depth distribution
TABLE 3

Measured Ionization Ratios

\begin{tabular}{|c|c|c|c|}
\hline Ratio & Data & $\mathrm{Sk}-67^{\circ} 111$ & AV 232 \\
\hline$\overline{N_{i}(\mathrm{~S} \text { IV }) / N_{i}(\mathrm{~S} \text { VI })}$ & FUSE I & $0.65 \pm 0.17^{\mathrm{a}}$ & $1.39 \pm 0.25$ \\
\hline$N_{i}(\mathrm{P}$ IV $) / N_{i}(\mathrm{P} \mathrm{v})$ & FUSE I & $0.59 \pm 0.21$ & $2.14 \pm 0.74$ \\
\hline$N_{i}(\mathrm{~N}$ III $) / N_{i}\left(\mathrm{~N} \mathrm{IV}^{\star}\right) \ldots \ldots$ & FUSE I & $2.09 \pm 1.17$ & $2.09 \pm 1.17$ \\
\hline$N_{i}\left(\mathrm{~N} \mathrm{IV}^{\star}\right) / N_{i}(\mathrm{~N} \mathrm{v}) \ldots \ldots$ & $I U E / \mathrm{FOS}$ & $0.12 \pm 0.03$ & $0.15 \pm 0.05$ \\
\hline
\end{tabular}

${ }^{a}$ Using $T=0.25$, which includes the narrow absorption component, $N_{i}(\mathrm{~S}$ IV $) / N_{i}(\mathrm{~S}$ VI $)=0.81 \pm 0.17$.

of ions through the wind is qualitatively similar in the two stars, once the difference in velocity is removed (right column, Figs. 1-3). This work demonstrates the unique capability afforded by the FUSE data to further our knowledge of hot star winds. First, the ability to measure different ionization stages of the same element allows us to constrain the ionization in the wind, thus refining mass-loss and element abundance determinations, which we will pursue in more complete modeling. Second, while the strong wind lines in the HST/IUE range (beyond $1200 \AA$ ) are usually saturated and yield only lower limits to optical depth estimates, lines from less abundant elements in the FUSE range have low optical depth, which can be measured with much greater precision by model fitting (Table 2).

L. B. is very grateful to Henny Lamers for enlightening discussions of the results and to Artemio Herrero for very useful suggestions. This work is based on data obtained for the Guaranteed Time Team by the NASA-CNES-CSA FUSE mission operated by Johns Hopkins University. Financial support to US participants has been provided by NASA contract NAS532985.

\section{REFERENCES}

Bianchi, L. 1982, in Third European IUE Conf., ed. E. Rolfe (ESA SP-176; Paris: ESA), 251

Bianchi, L., Hutchings, J. B., \& Massey, P. 1996, AJ, 111, 2303

Bianchi, L., Lamers, H., Hutchings, J., Massey, P., Kudritzki, R., Herrero, A., \& Lennon, D. 1994, A\&A, 292, 213

Bianchi, L., \& Scuderi, S. 1999, Mem. Soc. Astron. Italiana, 70, 667

Bianchi, L., Vassiliadis, E., \& Dopita, M. 1997, ApJ, 480, 290

Chlebowski, T., \& Garmany, C. D. 1991, ApJ, 368, 241

Chlebowski, T., Harnden, F. R., Jr., \& Sciortino, S. 1989, ApJ, 341, 427

Davidsen, A. F., et al. 1992, ApJ, 392, 264

Fitzpatrick, E. 1988, ApJ, 335, 703

Froese-Fischer, C., \& Godefroid, M. 1982, Nucl. Instrum. Methods, 202, 307

Haser, S., Lennon, D. J., Kudritzki, R.-P., Puls, J., Walborn, N. R., Bianchi, L., \& Hutchings, J. B. 1994, Space Sci. Rev., 66, 179

Hutchings, J. B. 1982, ApJ, 255, 70

Lamers, H., Cerruti-Sola, M., \& Perinotto, M. 1987, ApJ, 314, 726
Massa, D., et al. 2000, ApJ, 538, L47

McCluskey, G. E., Kondo, Y., \& Morton, D. C. 1975, ApJ, 201, 607

Moos, H. W., et al. 2000, ApJ, 538, L1

Morton, D. C. 1991, ApJS, 77, 119

Patriarchi, P., \& Perinotto, M. 1992, A\&A, 258, 285

Prinja, R., \& Crowther, P. A. 1998, MNRAS, 300, 828

Reistad, N., \& Engstrom, L. 1989, Phys. Rev. A, 39, 4518

Snow, T. P., \& Morton, D. C. 1976, ApJS, 32, 429

Walborn, N. R., Lennon, D., Haser, S., Kudritzki, R.-P., \& Voels, S. A. 1995a, PASP, 107, 104

Walborn, N. R., Long, K. S., Lennon, D. J., \& Kudritzki, R.-P. 1995b, ApJ, 454, L27

Wiese, W. L., Fuhr, J. R., \& Deters, T. M., eds. 1996, Atomic Transition Probabilities of Carbon, Nitrogen, and Oxygen: A Critical Data Compilation (Washington, DC: American Chemical Society) 